\title{
Validación por juicio de expertos de un instrumento de evaluación para evidencias de aprendizaje conceptual
}

\section{Validation by Expert Judment of an Evaluation Instrument for Evidence of Conceptual Learning}

Validação por julgamento de especialistas de um instrumento de avaliação para evidências de aprendizagem conceitual

Manuel Arnoldo Rodríguez Medina

Tecnológico Nacional de México, Campus Cd. Juárez, México manuel_rodriguez_itcj@yahoo.com https://orcid.org/0000-0002-8922-4718

Eduardo Rafael Poblano-Ojinaga Tecnológico Nacional de México, Campus La Laguna, México pooe_65@hotmail.com https://orcid.org/0000-0003-3482-7252

Lizette Alvarado Tarango Tecnológico Nacional de México, Campus Cd. Juárez, México lalvarado@itcj.edu.mx https://orcid.org/0000-0001-7934-8330

Arturo González Torres Tecnológico Nacional de México, Campus Milpa Alta, México cann.azteca13@gmail.com https://orcid.org/0000-0002-3337-7600

Manuel Iván Rodríguez Borbón Universidad Autónoma de Cd. Juárez, México ivan.rodriguez@uacj.mx https://orcid.org/0000-0001-8405-4599 


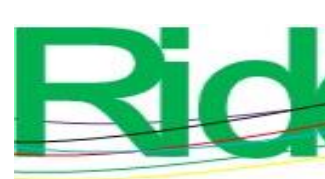
Revista Iberoamericana para la
Investigación y el Desarrollo Educativo
ISSN $2007-7467$

\section{Resumen}

Este trabajo de investigación educativa se centra en la validación de contenidos por juicio de expertos de un instrumento de evaluación para evidencias de aprendizajes conceptuales, específicamente la síntesis. Para el diseño del instrumento de evaluación, denominado lista de cotejo-síntesis, se identificaron los criterios y condiciones de calidad que debe contener una síntesis, elaborando un primer borrador alineado al estándar de competencia laboral EC0072. Posteriormente, un grupo de seis docentes conocedores del tema evaluaron la lista de cotejosíntesis según cuatro criterios: calidad, suficiencia, coherencia y relevancia. Los resultados empíricos reflejan que a 90 \% de los ítems evaluados se les asignó una calificación de cuatro, valor máximo en una escala de uno a cuatro. El análisis estadístico (prueba de Friedman) confirma el acuerdo o concordancia entre los jueces en tres de los cuatro criterios, por lo que el instrumento de evaluación fue revisado y mejorado en el criterio no concordante (claridad).

Palabras clave: instrumento de evaluación, juicio de expertos, validez de contenido.

\section{Abstract}

This educational research work focuses on the validation of contents by expert judgment of an evaluation instrument for evidence of conceptual learning, specifically the Synthesis. For the design of the evaluation instrument, called Checklist-Synthesis, the criteria and quality conditions that a Synthesis must contain were identified, preparing a first draft aligned to the EC0072 labor competence standard. Subsequently, a group of six knowledgeable teachers evaluate the Synthesis Checklist on four criteria: Quality, Sufficiency, Coherence and Relevance. The empirical results reflect that ninety percent of the items evaluated were assigned a rating of four, the maximum value on a scale of one to four. Statistical analysis (Friedman test) confirms the agreement between the judges in three of the four criteria, so the evaluation instrument was revised and improved in the non-concordant criterion - Clarity.

Keywords: Content Validity, Evaluation Instrument. Expert Judgment. 

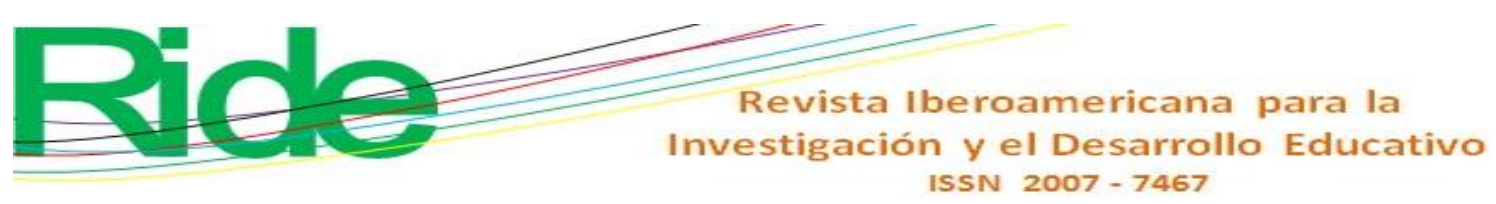

\section{Resumo}

Este trabalho de pesquisa educacional centra-se na validação de conteúdo por julgamento de especialistas de um instrumento de avaliação para evidências de aprendizagem conceitual, especificamente síntese. Para a concepção do instrumento de avaliação, denominado checklistsíntese, foram identificados os critérios e condições de qualidade que uma síntese deve conter, elaborando um primeiro esboço alinhado à norma de competência laboral EC0072. Posteriormente, um grupo de seis professores experientes avaliou o checklist-síntese segundo quatro critérios: qualidade, suficiência, coerência e relevância. Os resultados empíricos refletem que $90 \%$ dos itens avaliados receberam nota quatro, valor máximo em uma escala de um a quatro. A análise estatística (teste de Friedman) confirma a concordância ou concordância entre os juízes em três dos quatro critérios, portanto o instrumento de avaliação foi revisado e aprimorado no critério não concordante (clareza).

Palavras-chave: instrumento de avaliação, julgamento de especialistas, validade de conteúdo. Fecha Recepción: Noviembre 2020

Fecha Aceptación: Mayo 2021

\section{Introducción}

En este trabajo se presenta la validación por juicio de expertos de un instrumento de evaluación (IE) del tipo lista de cotejo, el cual fue usado para determinar una evidencia de aprendizaje conceptual en torno a una síntesis, entendida esta como la exposición abreviada sobre un tema que debe ser explicado de forma personal por el alumno para facilitar su comprensión.

Dado que la medición es el proceso que vincula conceptos abstractos (variable latente o constructo supuesto), los cuales solo pueden ser medidos mediante variables observables (Cupani, 2012), cualquier instrumento de medición o recopilación de datos debe cumplir tres requisitos esenciales: objetividad, validez y confiabilidad (Hernández, Fernández y Batista, 2014).

La objetividad se refiere al grado en que el instrumento es permeable a la influencia de los prejuicios y tendencias del investigador que lo administra, califica e interpreta, y se refuerza con la estandarización en la aplicación del instrumento (iguales instrucciones y condiciones para todos los participantes) y en la evaluación de los resultados; así como empleando personal capacitado y experimentado en el instrumento. 

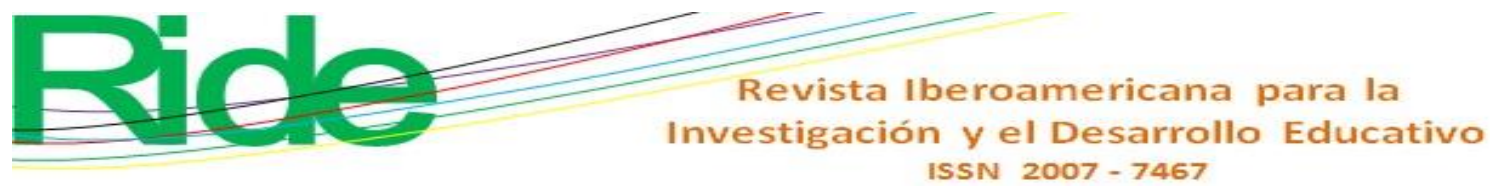

La validez es el grado en que un instrumento mide realmente la variable que se quiere medir, y en la literatura se identifican tres tipos principales: contenido, criterio y construcción. La validez del contenido por juicio experto es la empleada en este trabajo, entendida como el grado en que un instrumento de medición aparentemente mide la variable en cuestión, de acuerdo con "voces calificadas".

La confiabilidad se refiere al grado en que su aplicación repetida al mismo individuo u objeto produce resultados iguales. Es importante mencionar que un instrumento de medición puede ser confiable, pero no necesariamente válido; por ello, es requisito que demuestre ser confiable y válido. De no ser así, los resultados no deben tomarse en serio (Hernández et al., 2014).

Ahora bien, en cuanto a la evaluación del aprendizaje con enfoque en competencias profesionales, vale destacar que debe ser objetiva porque integra un conjunto de evidencias que pueden confirmar el alcance de la competencia por el estudiante, es decir, debe basarse en evidencias producidas en las actividades de aprendizaje determinadas por el profesor. Por lo tanto, la evaluación de las competencias es un proceso integral, permanente, sistemático y objetivo que debe servir para determinar si se han alcanzado las metas establecidas en la asignatura (Tecnológico Nacional de México [TecNM], 2015, 2018).

El profesor, por ende, debe considerar la integración de información cuantitativa y cualitativa, así como los diferentes tipos y formas de la evaluación y una diversidad de instrumentos, de tal manera que los corresponsables del proceso puedan tomar decisiones oportunas en busca de una mejora permanente.

En este sentido, en el Tecnológico Nacional de México (TecNM), para la evaluación de las evidencias (producto del aprendizaje conceptual, actitudinal y procedimental), se recomienda el uso de instrumentos de evaluación. El estándar de competencia EC0772 Evaluación del aprendizaje con un enfoque en habilidades profesionales establece como requisito de certificación que el maestro del TecNM sea capaz de diseñar y aplicar al menos cuatro instrumentos de evaluación: la lista de cotejo, la guía de observación, el cuestionario y la rúbrica. De hecho, también indica las características generales de estos IE.

Se supone que si el profesor utiliza un instrumento para evaluar estas evidencias (donde se indiquen los requisitos/condiciones de calidad esperadas del alumno), el estudiante sabrá con certeza cuáles son las exigencias que debe cumplir, por lo que podrá diseñar líneas de acción para alcanzar tal fin. 

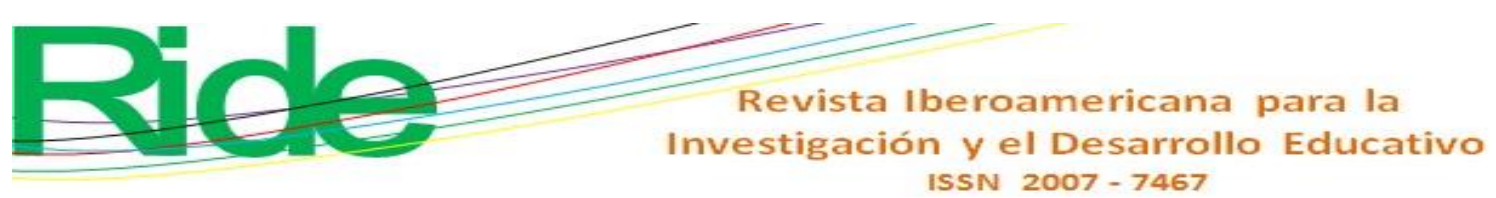

Dicho esto, para la validación por juicio de expertos se utilizan pruebas no paramétricas como la de Friedman (análisis no paramétrico de un experimento de bloques aleatorizado), la cual ofrece una alternativa al análisis de varianza de dos factores (Granato, De Araújo Calado y Jarvis, 2014). Los experimentos de bloques aleatorizados son una generalización de experimentos pareados, y la prueba de Friedman es una generalización de prueba de signos pareados.

\section{Metodología}

La metodología empleada fue cuantitativa porque se utilizó la recolección de datos para probar hipótesis con base en la medición numérica y el análisis estadístico, realizados por medio de un conjunto de procesos de investigación que implicaron la recolección y el análisis de datos cuantitativos, así como su integración y discusión, con el propósito de lograr un mayor entendimiento del fenómeno estudiado mediante inferencias de los resultados de la información recolectada (Bernal, 2010; Hernández et al., 2014; Malhotra, 2008).

\section{Materiales}

En la etapa de revisión de literatura, el principal insumo fueron los artículos y las publicaciones identificadas en la Biblioteca Virtual (BIVIR) de la Universidad Autónoma de Ciudad Juárez, la cual cuenta con 30 base de datos, entre ellas Anual Review, Ebsco, Elsevier, Emerald, Sciencedirect, Wiley, etc.

Para evaluar el contenido y estructura de la síntesis fue necesario diseñar un IE tipo lista de cotejo, diseñado a partir de la revisión de la literatura para que cumpliera con las condiciones de calidad del estándar de competencia laboral EC0772. Los análisis estadísticos de datos se llevarán a cabo en el paquete computacional Minitab, versión 17.

\section{Método}

Siguiendo una dinámica similar a la propuesta por Romero, Gómez y Parroquín (2016), Romero, Matheus-Mari y Poblano-Ojinaga (2017) y Poblano-Ojinaga, López, Gómez y TorresArguelles (2019), esta investigación ha sido planificada en tres pasos: en el primero — dado que la medición es un proceso de vincular conceptos abstractos con indicadores empíricos para registrar información o datos sobre las variables, y debido a que un IE registra datos observables que realmente representan los conceptos o variables en estudio- se procedió a identificar los 


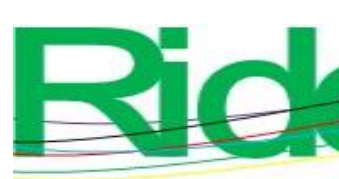

\section{Revista Iberoamericana para la Investigación y el Desarrollo Educativo ISSN 2007 - 7467}

criterios y las condiciones de calidad que debe cumplir una síntesis a través de una revisión de la literatura y mediante la opinión de dos expertos en el tema. De este modo se consiguió un primer borrador de lista de criterios y condiciones de calidad que debe contener el IE.

En el segundo paso (diseño del instrumento de evaluación), con base en la información obtenida en el punto 1 y considerando la estructura requerida en el elemento 4 de EC0772, se definieron los requisitos o condiciones de calidad que debe contener una síntesis, lo que sirvió para preparar un borrador de la lista de cotejo.

El tercer paso fue la validación del contenido a través del juicio de expertos del IE, para lo cual se solicitó a un grupo de expertos que evaluaran la lista de cotejo en cuatro áreas (suficiencia, claridad, coherencia y relevancia), siguiendo el procedimiento recomendado por Escobar-Pérez y Cuervo-Martínez (2008).

Para la validación por juicio de expertos se utilizaron pruebas no paramétricas porque se usaron datos nominales, categóricos y ordinales. Asimismo, seleccionamos la prueba de Friedman para determinar las diferencias en la ubicación central (mediana) para el análisis de ensayos con medidas repetidas unidireccionales que tienen tres o más muestras dependientes (Granato et al., 2014); en este caso, para determinar el grado de acuerdo entre los expertos y su valor $\boldsymbol{p}$ para elegir entre dos hipótesis opuestas en función de sus datos recopilados:

$\mathrm{H}_{0}$ : No existe un acuerdo significativo entre los conocedores/jueces.

$\mathrm{H}_{1}$ : Existe un acuerdo significativo entre los conocedores/jueces.

\section{Resultados}

En el primer paso se identificaron los criterios y las condiciones de calidad o aspectos a considerar (Beltrán, 2005; Fernández y Bressia, 2009; Quispe y Melanez, 2018) para evaluar una síntesis presentada por los estudiantes como evidencia de una actividad de aprendizaje conceptual. Asimismo, se les solicitó a dos docentes (decanos del Departamento EconómicoAdministrativo del campus La Laguna) la revisión de la lista de condiciones de calidad o criterios de evaluación de la síntesis, así como sus recomendaciones y sugerencias. El resultado de esta etapa fue un primer borrador de la lista de cotejo que incluyó cinco criterios y diez condiciones de calidad (tabla 1). 

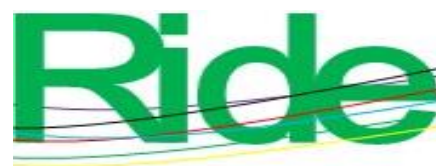

Tabla 1. Lista de criterios y condiciones de calidad para la lista de cotejo-síntesis

\begin{tabular}{|c|c|c|}
\hline \multirow{4}{*}{ Forma } & Portada & Nombre del alumno, tema y fecha de elaboración. \\
\hline & \multirow{3}{*}{ Presentación } & 5-7 páginas a renglón sencillo. \\
\hline & & Clara y fácil de entender. \\
\hline & & $\begin{array}{l}\text { Con sus propias palabras y sin errores de } \\
\text { ortografía. }\end{array}$ \\
\hline \multirow{6}{*}{ Fondo } & Introducción & $\begin{array}{l}\text { Breve explicación del tema. Idea principal e } \\
\text { importancia. }\end{array}$ \\
\hline & \multirow{3}{*}{ Desarrollo } & La idea principal \\
\hline & & Los puntos que giran alrededor de la idea principal. \\
\hline & & Los beneficios y áreas de aplicación (si aplica). \\
\hline & \multirow[t]{2}{*}{ Conclusión } & $\begin{array}{l}\text { Conclusión central del tema (incluye palabras y } \\
\text { frases claves). }\end{array}$ \\
\hline & & Aportación-juicio-opinión. \\
\hline
\end{tabular}

Fuente: Elaboración propia

En el paso dos (diseño del IE), con la finalidad de elaborar el primer borrador de la lista de cotejo-síntesis, se tomaron en cuenta los comentarios y cambios propuestos por los decanos, así como la estructura requerida en el elemento 4 del EC0772. Los criterios de desempeño se enlistan a continuación:

- Los instrumentos de evaluación basados en la actividad de enseñanza-aprendizaje y la competencia elaborados: corresponden al nivel de desempeño de las competencias.

- La lista de verificación preparada incluye reactivos que cumplen con la siguiente estructura: artículo, objeto, verbo y condición de calidad; corresponde al tipo de evidencia a evaluar (Consejo Nacional de Normalización y Certificación de Competencias Laborales [Conocer], 2016).

El resultado de esta etapa fue el instrumento presentado a los conocedores o expertos para su evaluación (figura 1). 


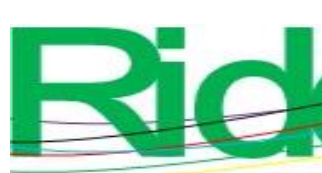

de

Revista Iberoamericana para la

Investigación y el Desarrollo Educativo

ISSN $2007-7467$

Figura 1. Instrumento para evaluación de la lista de cotejo-síntesis por juicio de expertos

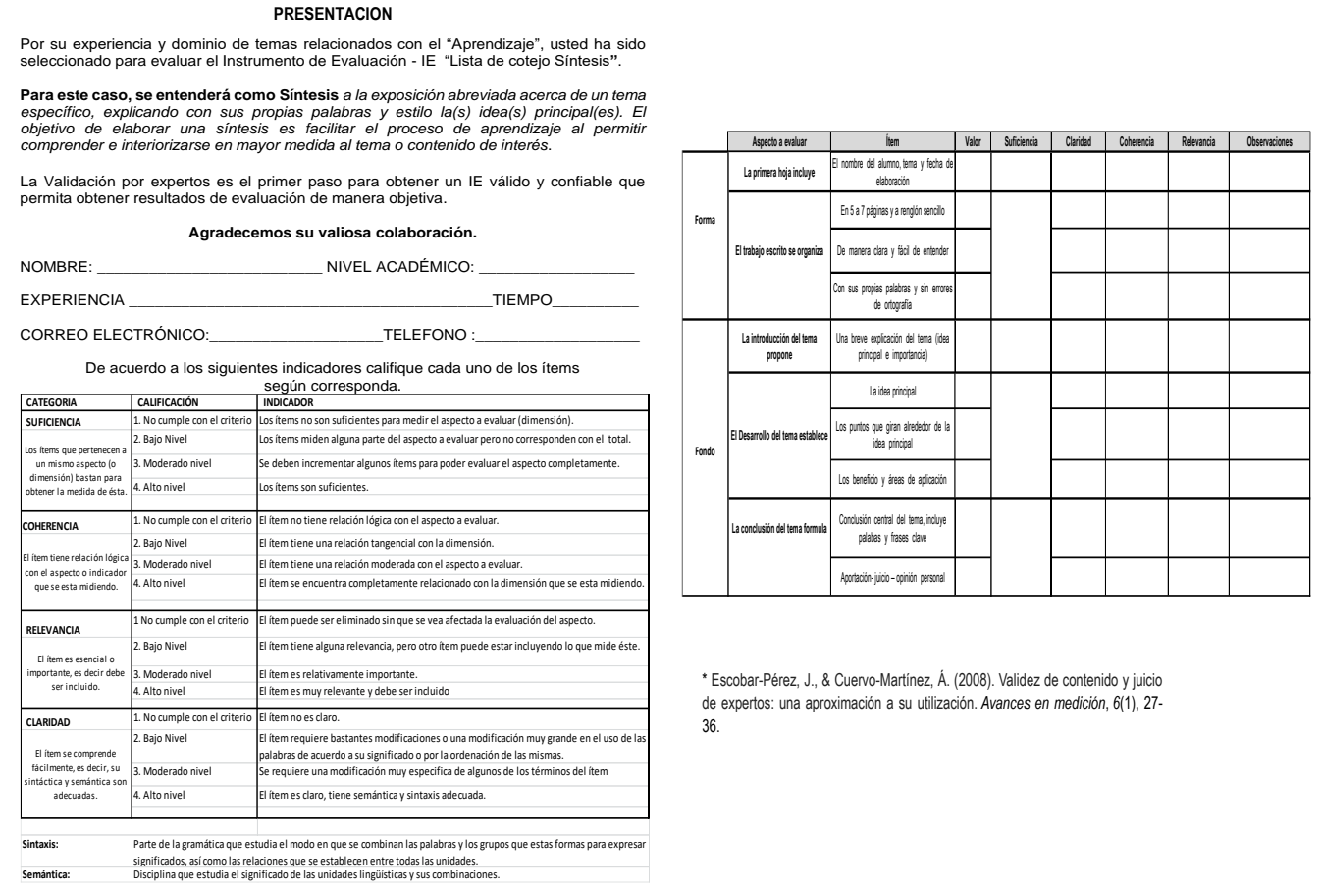

Fuente: Elaboración propia

En el paso tres (validación del IE por expertos), se solicitó a un grupo de 10 expertos (docentes con amplia experiencia) que evaluaran la lista de cotejo-síntesis en 4 diferentes áreas: suficiencia, relevancia, claridad y coherencia. Aquí se eliminaron 4 evaluaciones por presentar inconsistencias.

La selección de los expertos o conocedores se basó en tres criterios: experiencia docente (al menos 15 años), formación académica (con grado de doctor en educación o área afín, preferentemente) y estar impartiendo cátedra en el momento del estudio (frente a grupo). De ese grupo de 10 docentes seleccionados, 4 evaluaciones se eliminaron por inconsistencias. Una exploración inicial de los resultados de las evaluaciones en los cuatro criterios evidenció que de las 210 asignaciones de un valor a los ítems/criterios, en $90 \%$ de las veces los evaluadores coinciden en asignar un valor de 4 al ítem, y $8.6 \%$ en asignarle un valor de 3, lo que de manera empírica demostraría el acuerdo entre los 6 jueces (figura 2). 
Figura 2. Resultados de la evaluación de los ítems por juicio de expertos

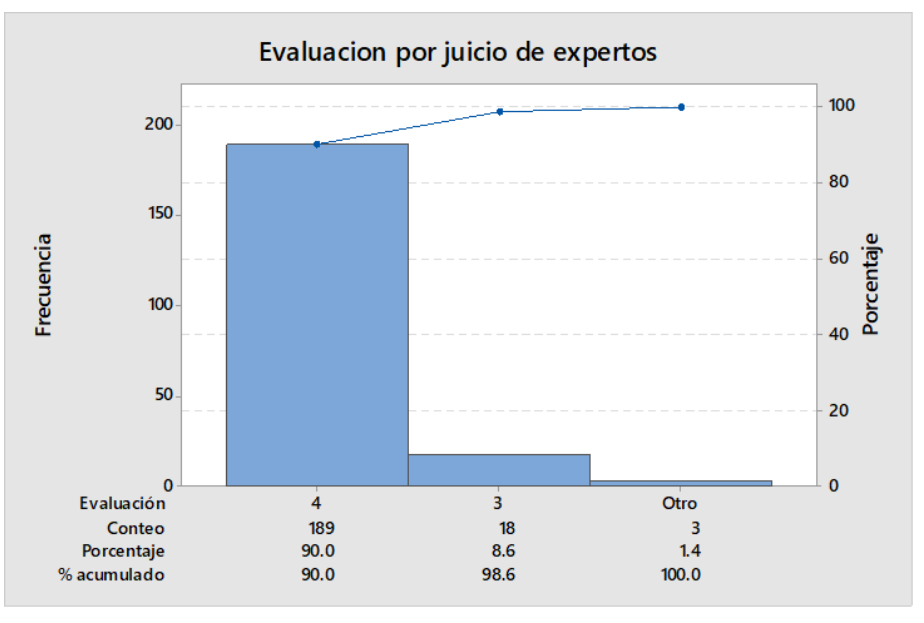

Fuente: Elaboración propia

A manera de ejemplo, en relación con el criterio claridad, los expertos asignaron a cada ítem un valor (entre 1 y 4) según consideraron correcto o conveniente (tabla 2).

Tabla 2. Resultado de la evaluación por expertos al criterio claridad

\begin{tabular}{|c|c|c|c|c|c|c|}
\hline \multicolumn{7}{|c|}{ Experto/conocedor } \\
\hline Ítem & 1 & 2 & 3 & 4 & 5 & 6 \\
\hline 1 & 4 & 4 & 4 & 4 & 4 & 4 \\
\hline 2 & 3 & 4 & 4 & 4 & 4 & 4 \\
\hline 3 & 4 & 4 & 4 & 4 & 4 & 4 \\
\hline 4 & 4 & 4 & 4 & 3 & 4 & 4 \\
\hline 5 & 3 & 4 & 4 & 3 & 4 & 4 \\
\hline 6 & 4 & 3 & 4 & 3 & 4 & 4 \\
\hline 7 & 3 & 4 & 4 & 3 & 4 & 4 \\
\hline 8 & 4 & 3 & 4 & 4 & 4 & 4 \\
\hline 9 & 4 & 4 & 4 & 4 & 4 & 4 \\
\hline 10 & 4 & 4 & 4 & 4 & 4 & 4 \\
\hline
\end{tabular}

Fuente: Elaboración propia

Posteriormente, los datos de la tabla 2 se analizaron estadísticamente con la ayuda del software Minitab, versión17, por medio de la prueba de Friedman. Las hipótesis planteadas fueron las siguientes: 
$\mathrm{H}_{0}$ : Todos los efectos del tratamiento son cero.

$\mathrm{H}_{1}$ : No todos los efectos del tratamiento son ceros.

Tabla 3. Resultado de la prueba de Friedman del criterio claridad (Minitab*)

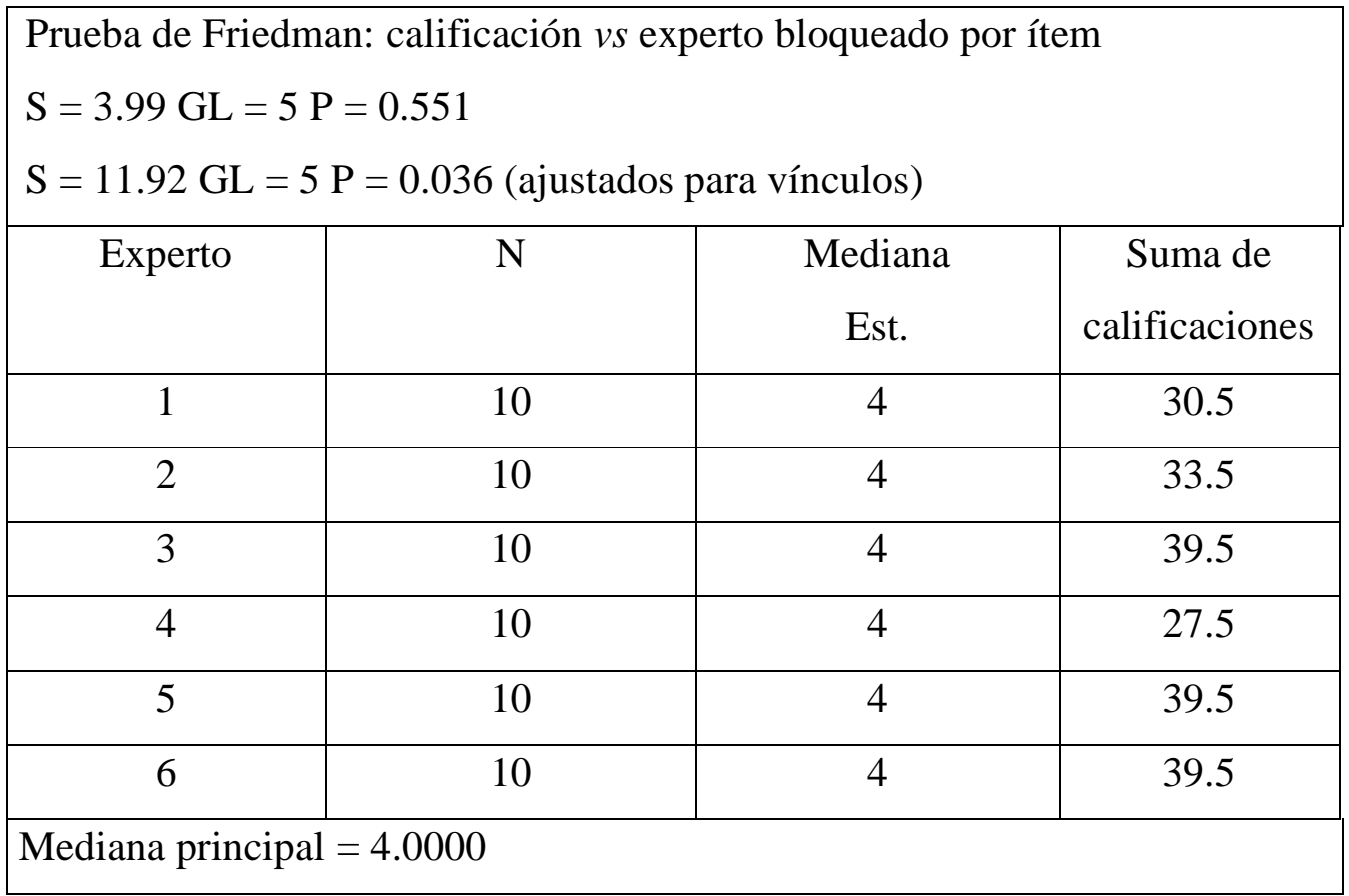

Fuente: Elaboración propia

* Minitab imprime la estadística de prueba, la cual tiene una distribución aproximadamente chicuadrada y los grados de libertad (número de tratamientos menos uno). Si existen empates dentro de uno o más bloques, se utiliza la clasificación promedio y se imprime también una estadística de prueba corregida para empates. Si hay muchos empates, la estadística de prueba no corregida es conservadora; la versión corregida es usualmente más cercana, pero puede ser conservadora o liberal, y muestra una mediana estimada para cada nivel de tratamiento. La mediana estimada es la mediana principal más el efecto del tratamiento.

De la misma manera, se procedió a llevar a cabo la prueba de Friedman para los criterios suficiencia, relevancia y coherencia. En la tabla 4 se presentan los resultados obtenidos para S (ajustado para vínculos). 

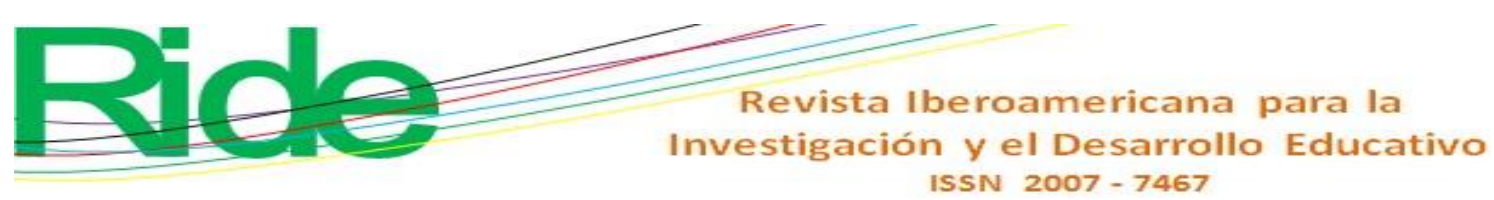

los criterios de las evidencias que presente el alumno no están formal y/o claramente definidos por el profesor o docente), este objetivo se cumple al contar con un IE 1 lista de cotejo-síntesis, formal y de fácil uso para los estudiantes y profesores, y debidamente validado por juicio de expertos.

Por otra parte, es importante mencionar que al momento de seleccionar a las personas que participarán en la evaluación del instrumento de medición/evaluación se debe prever que conozcan el tema a tratar, ya sea por experiencia en el campo laboral, por su formación profesional o por su trayectoria académica. También se recomienda anticipar la cantidad de expertos que fungirán como jueces, de acuerdo con las características de la prueba y el análisis estadístico.

\section{Futuras Líneas de Investigación}

Este trabajo es la primera etapa del proyecto de investigación educativa Diseño y validación de un instrumento de evaluación IE para aprendizajes conceptuales basados en lineamientos del TecNm y el estándar EC0072, y su efecto en los índices de reprobación (ITFLLAG-PIE-2019-0228). Por lo tanto, la siguiente etapa se enfocará en la evaluación de la consistencia interna (confiabilidad) del instrumento por medio del alfa de Conbrach a través de una corrida piloto en diferentes grupos.

Una limitación de este estudio fue que la validación por juicio de expertos del IE se llevó a cabo en una institución de educación superior perteneciente al Tecnológico Nacional de México, por lo que los resultados no pueden ser generalizados; sin embargo, dado que se presenta de manera clara y concisa el método de validación, el procedimiento podría ser útil para otros instrumentos, como cuestionarios, rúbricas, etc.

\section{Agradecimientos}

Un agradecimiento al MC. José Luis Zapata Dávila, director del TecNM campus La laguna, por su apoyo en el registro y en la realización de este proyecto de investigación educativa; asimismo, a los docentes Dra. Diana Margarita Vázquez Peña y Dr. Ricardo Coronado Velasco, decanos del Departamento Económico-Administrativo; a la Dra. Ma. del Rosario Mayorga, al Dr. Armando Longoria Gándara, al Dr. Armando Longoria de la Torre, al Dr. José Ruiz Ayala, y al M.E. Alejandro Romero Jiménez por su participación en la evaluación por juicio de expertos. 


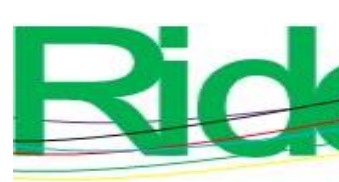

\section{Referencias}

Beltrán, O. A. (2005). Revisiones sistemáticas de la literatura. Revista Colombiana de Gastroenterología, 20(1), 60-69.

Bernal, C. (2010). Metodología de la investigación (3. a ed.). Colombia: Pearson Educación.

Consejo Nacional de Normalización y Certificación de Competencias Laborales (Conocer) (2016). Estándar de competencia EC0772. Evaluación del aprendizaje con enfoque en competencias profesionales. Comité de Gestión de Competencias del Tecnológico Nacional de México Conocer.

Cupani, M. (2012). Análisis de ecuaciones estructurales: conceptos, etapas de desarrollo y un ejemplo de aplicación. Revista Tesis, 2(1), 186-199.

Escobar-Pérez, J. y Cuervo-Martínez, A. (2008). Validez de contenido y juicio de expertos: una aproximación a su utilización. Avances en Medición, 6, 27-36.

Fernández, L. y Bressia, R. (2009). Definiciones y características de los principales tipos de texto. Buenos Aires, Argentina. Recuperado de http://www.uca.edu.ar/uca/common/grupo18/files/Definicion_generos_discursivos_ abril_2009.pdf

Granato, D., De Araújo Calado, V. M. and Jarvis, B. (2014). Observations on the use of statistical methods in food science and technology. Food Research International, 55, 137-149.

Hernández, R., Fernández, C. y Batista, P. (2014). Metodología de la investigación (6. ${ }^{a}$ ed.). México: Editorial Mc Graw Hill Education.

Malhotra, N. (2008). Investigación de mercados (5. ${ }^{\mathrm{a}}$ ed.). México: Editorial Pearson.

Poblano-Ojinaga, E., López, R., Gómez, J. and Torres-Arguelles, V. (2019). Effect of competitive intelligence on innovation capability: An exploratory study in Mexican companies. Journal of Intelligence Studies in Business, 9(3) 62-67.

Quispe, C. y Melanez, Y. (2018). La síntesis como estrategia para la comprensión de textos literarios en el área de comunicación en los estudiantes del cuarto grado de la Institución Secundaria César Vallejo de Juliaca durante el año 2018 (tesis de licenciatura). Perú: Universidad Nacional del Altiplano. Recuperado de http://repositorio.unap.edu.pe/bitstream/handle/UNAP/9226/Quispe_P\%C3\%A9rez_Cl ever_Ticona_Quispe_Yoli\%C3\%B1o_Melanez.pdf?sequence=1 


\begin{tabular}{|c|c|}
\hline Rol de Contribución & Autor(es) \\
\hline Conceptualización & $\begin{array}{l}\text { Manuel Arnoldo Rodríguez Medina (principal), Eduardo } \\
\text { Rafael Poblano Ojinaga (igual). }\end{array}$ \\
\hline Metodología & $\begin{array}{l}\text { Eduardo Rafael Poblano Ojinaga (principal), Manuel Iván } \\
\text { Rodríguez Borbón (que apoya). }\end{array}$ \\
\hline Software & $\begin{array}{l}\text { Manuel Arnoldo Rodríguez Medina (principal), Lizette } \\
\text { Alvarado Tarango (que apoya), Manual Iván Rodríguez } \\
\text { Borbón (que apoya). }\end{array}$ \\
\hline Validación & $\begin{array}{l}\text { Eduardo Rafael Poblano Ojinaga (principal), Lizette Alvarado } \\
\text { Tarango (que apoya), Arturo González Torres (que apoya). }\end{array}$ \\
\hline Análisis Formal & $\begin{array}{l}\text { Manuel Arnoldo Rodríguez Medina (principal), Eduardo } \\
\text { Rafael Poblano Ojinaga (igual). }\end{array}$ \\
\hline Investigación & $\begin{array}{l}\text { Eduardo Rafael Poblano Ojinaga (principal), Lizette Alvarado } \\
\text { Tarango (igual), Arturo González Torres (que apoya). }\end{array}$ \\
\hline Recursos & $\begin{array}{l}\text { Manuel Arnoldo Rodríguez Medina (principal), Lizette } \\
\text { Alvarado Tarango (que apoya). }\end{array}$ \\
\hline Curación de datos & $\begin{array}{l}\text { Manuel Arnoldo Rodríguez Medina (principal), Eduardo } \\
\text { Rafael Poblano Ojinaga (igual), Manuel Iván Rodríguez } \\
\text { Borbón (que apoya). }\end{array}$ \\
\hline $\begin{array}{l}\text { Escritura - Preparación del } \\
\text { borrador original }\end{array}$ & $\begin{array}{l}\text { Lizette Alvarado Tarango (principal), Arturo González Torres } \\
\text { (igual), Manuel Iván Rodríguez Borbón (que apoya). }\end{array}$ \\
\hline $\begin{array}{l}\text { Escritura - Revisión y } \\
\text { edición }\end{array}$ & $\begin{array}{l}\text { Manuel Arnoldo Rodríguez Medina (principal), Eduardo } \\
\text { Rafael Poblano Ojinaga (igual). Arturo González Torres (que } \\
\text { apoya). }\end{array}$ \\
\hline Visualización & $\begin{array}{l}\text { Manuel Arnoldo Rodríguez Medina (principal), Eduardo } \\
\text { Rafael Poblano Ojinaga (igual). }\end{array}$ \\
\hline Supervisión & $\begin{array}{l}\text { Manuel Arnoldo Rodríguez Medina (principal), Eduardo } \\
\text { Rafael Poblano Ojinaga (igual), Lizette Alvarado Tarango (que } \\
\text { apoya). }\end{array}$ \\
\hline Administración de Proyectos & $\begin{array}{l}\text { Eduardo Rafael Poblano Ojinaga (principal), Arturo González } \\
\text { Torres (igual), Manuel Iván Rodríguez Borbón (que apoya). }\end{array}$ \\
\hline Adquisición de fondos & $\begin{array}{l}\text { Manuel Arnoldo Rodríguez Medina (principal), Lizette } \\
\text { Alvarado Tarango (igual). }\end{array}$ \\
\hline
\end{tabular}




\section{Anexo}

\section{Lista de cotejo-síntesis}

Departamento de Ingeniería Industrial - TecNM / Campus La Laguna

Asignatura: “

\begin{tabular}{|l|l|l|}
\hline Nombre del alumno: & \multicolumn{2}{|l|}{} \\
\hline Carrera: & $\begin{array}{l}\text { INGENIERIA INDUSTRIAL } \\
\text { PROFESOR: CLAVE:___, GRUPO: }\end{array}$ \\
\hline No. Control: & & Fecha: \\
\hline
\end{tabular}

Instrucciones para el evaluador: Marque con una " $\sqrt{ }$ " en la columna "Cumple" cuando la Síntesis presentada por el estudiante muestra la evidencia de los apartados correspondientes y en caso contrario deje la casilla sin llenar.

Lista de cotejo Síntesis \# __ Tema:

\begin{tabular}{|c|l|c|l|c|}
\hline No. & \multicolumn{1}{|c|}{ Aspecto a evaluar } & Valor & Cumple & Obs. \\
\hline 1 & La primera hoja incluye & & & \\
\hline${ }^{*}$ & El nombre del alumno, tema y fecha de elaboración & 5 & & \\
\hline 2 & El trabajo escrito se organiza & & & \\
\hline${ }^{*}$ & En 5 a 7 páginas y a renglón sencillo & 5 & & \\
\hline$*$ & De manera clara y fácil de entender & 10 & & \\
\hline${ }^{*}$ & Con sus propias palabras y sin errores de ortografía & 15 & & \\
\hline 3 & La introducción del tema propone & & & \\
\hline${ }^{*}$ & Una breve explicación del tema (idea principal e importancia) & 15 & & \\
\hline 4 & El Desarrollo del tema establece & & & \\
\hline$*$ & La idea principal & 15 & & \\
\hline$*$ & Los puntos que giran alrededor de la idea principal & 10 & & \\
\hline$*$ & Los Beneficio y áreas de aplicación & 5 & & \\
\hline 5 & La conclusión formula & & & \\
\hline$*$ & Conclusión central del tema, incluye palabas y frases clave & 10 & & \\
\hline$*$ & Aportación- juicio - opinión personal & 10 & & \\
\hline & & & Total & \\
\hline
\end{tabular}

Síntesis: Exposición abreviada acerca de un tema especifico, explicando con sus propias palabras y estilo la(s) idea(s) principal(es). El objetivo de elaborar una síntesis es facilitar el proceso de aprendizaje al permitir comprender e interiorizarse en mayor medida al tema o contenido de interés.

\begin{tabular}{|l|}
\hline RETROALIMENTACION \\
\hline Aspectos positivos: \\
1.- \\
2.- \\
3.- \\
4.- \\
5.- \\
\hline Áreas / Sugerencias de mejora: \\
1.- \\
2.- \\
3.- \\
4.- \\
5.- \\
\hline AsporTUNIDAD \\
1.- \\
2.- \\
3.- \\
4.- \\
5.- \\
\hline Áreas / Sugerencias de mejora: \\
1.- \\
2.- \\
3.- \\
4.- \\
5.- \\
\hline Nombre y firma del(os) alumno(s): \\
\hline
\end{tabular}

Nivel de desempeño aceptable: 70 pts.

Fuente: Elaboración propia 